\title{
La metodología de opciones reales: una aplicación al caso de una empresa papelera de la región del Biobío, Chile
}

\author{
The methodology of real options: an application to the case \\ of a paper company in the Biobio region, Chile \\ Rodrigo Ahumada V. ${ }^{1} \quad$ Alejandro Andalaft Ch. ${ }^{1}$ \\ Recibido 11 de abril de 2012, aceptado 9 de mayo de 2013 \\ Received: April 11, 2012 Accepted: May 9, 2013
}

\begin{abstract}
RESUMEN
En el presente artículo se evalúa económicamente la construcción de una nueva planta de cogeneración de energía limpia con biomasa forestal por parte de la empresa Norske Skog Biobío (NSBB). Esto para demostrar que esta central a base de biomasa forestal para generación de electricidad es una opción viable en Chile desde la perspectiva económica y ambiental. Para esta evaluación se propone un enfoque que utiliza la "teoría de opciones reales", basada en la aplicación del Método Binomial con Transformada Logarítmica en seis pasos. Además se comparan los resultados obtenidos con este enfoque con los resultados usando los métodos tradicionales. Se comprueba que para todos los casos de estudio el valor arrojado por el método de opciones reales fue mayor al calculado mediante métodos tradicionales. Se concluye entonces que esta alternativa de inversión resulta rentable.
\end{abstract}

Palabras clave: Metodología de opciones reales, cogeneración, fábrica de papel, biomasa.

\begin{abstract}
This paper evaluates economically build a new cogeneration plant with clean energy from forest biomass company Norske Skog Bio Bio (NSBB). This is to show that this plant-based forest biomass for electricity generation is a viable option in Chile from the economic and environmental perspective. For this evaluation, we propose an approach that uses "real options theory" based on the application of log-transformed binomial method with six steps. Also, comparing the results obtained with this approach, the results using traditional methods. It is found that for all cases studied, the value yielded by the real options method was higher than estimated by traditional methods. It is concluded that this alternative profitable investment.
\end{abstract}

Keywords: Real options methodology, cogeneration, paper mill, biomass.

\section{INTRODUCCIÓN}

Chile en las próximas décadas aumentará su vulnerabilidad al cambio climático. El principal efecto que se prevé es la disminución de precipitaciones en gran parte del valle central. Esto tenderá a aumentar la dependencia en combustibles fósiles, lo que implicará alzas en las tarifas a menos que se privilegien y potencien la eficiencia energética (EE) y las ERNC.
El supuesto principal de este escenario "verde"es que el gobierno adopte una política proactiva para la EE y las ERNC. Si se fija como meta nacional un $20 \%$ de renovables hacia el 2020, es indispensable esta acción. El hacerlo significa lograr a 4.400 MW (estimación de cálculo de energías verdes, como eólica, biomasa, minihidro, geotermia y solar) de potencia con una generación de 20.147 GWh al 2025. Si a lo anterior sumamos un ahorro de 22.313 GWh al 2025 lograble por EE, está claro que el

\footnotetext{
1 Departamento de Ingeniería Industrial. Facultad de Ingeniería. Universidad de Concepción. Concepción, Chile. E-mail: rahumada@indap.cl; aandalaf@udec.cl
} 
margen real es muy superior y que Hidroaysén no es en absoluto necesario.

La región del Biobío puede ser un aporte a esta matriz energética verde por medio de su biomasa forestal para alcanzar los $600 \mathrm{MW}$ como país al año 2025. Esta energía se puede generar del bosque nativo con una superficie de 785.766 ha (equivale al $6 \%$ del país) y plantaciones forestales con una superficie de 791.831 ha (equivale al $38 \%$ del país) concentrada en esta región. Adicionalmente, existe un potencial bruto para generar electricidad (MW) tanto de la industria forestal (residuos) como del manejo de plantaciones (bosque nativo y bosque forestal).

Por ende, la sumatoria de ambas fuentes para la combustión directa y cogeneración (electricidad y vapor) en esta región puede alcanzar un potencial bruto considerando un intervalo mínimo de 472 (MW) y un máximo de 1.007 (MW), que equivale a $16 \%$ del país. Sin embargo, el potencial bruto factible (potencial bruto (MW) x nivel de factibilidad) considerando solo manejo forestal (pino, eucaliptos), residuos industriales forestales y madera y manejo de bosque nativo (áreas comerciales), puede alcanzar una capacidad mínima de 94 (MW) y máxima de 200 (MW).

El artículo se centra en una evaluación económica de un proyecto de cogeneración de electricidad con biomasa forestal para la empresa papelera NSBB, basada en la metodología de opciones reales. Ello debido a que este tipo de empresa tiene un uso intensivo de electricidad y vapor convencional en el proceso de fabricación de papel, que influye en los costos de producción de papel. Por ejemplo, la participación de la generación hidroeléctrica en el Sistema Interconectado Central (SIC), ha resultado en situaciones muy críticas cada vez que se producen sequías y, por ende, aumento en el precio nudo de electricidad. Adicionalmente se observa un mercado altamente concentrado, con solo cinco empresas que generan a lo menos el $83 \%$ de electricidad (Tabla 1).

Lo anterior se podría superar con un mayor número de actores que participen en este mercado, cuya principal fuente de aporte son las energías renovables no convencionales (ERNC; energías verdes), sobre todo en esta región por la disponibilidad de biomasa forestal y potencial bruto factible de generación de
Tabla 1. Participación de mercado por empresa en el SIC

\begin{tabular}{|l|c|c|}
\hline $\begin{array}{c}\text { Empresas } \\
\text { generadoras }\end{array}$ & $\begin{array}{c}\text { \% } \\
\text { Participación } \\
\text { de mercado }\end{array}$ & $\begin{array}{c}\text { \% } \\
\text { Concentración }\end{array}$ \\
\cline { 1 - 2 } Endesa & $40 \%$ & \multirow{2}{*}{8} \\
\cline { 1 - 2 } Colbún & $21 \%$ & \\
\cline { 1 - 2 } AES Gener & $14 \%$ & \\
\hline E.E. Guacolda & $5 \%$ & \\
\hline Pacific Hidro & $3 \%$ & $17 \%$ \\
\hline Otros & $17 \%$ & $\mathbf{1 0 0 \%}$ \\
\hline Total & $\mathbf{1 0 0 \%}$ & \\
\hline
\end{tabular}

Fuente: Universidad Técnica Federico Santa María [1].

Tabla 2. Potencial bruto por fuente de biomasa (en MW).

\begin{tabular}{|c|c|c|c|}
\hline Fuente Biomasa & Min. & Máx. & \% \\
\hline $\begin{array}{c}\text { Manejo Forestal } \\
\text { (pino, eucaliptus) }\end{array}$ & 152 & 203 & $24 \%$ \\
\hline $\begin{array}{c}\text { Residuos Industriales } \\
\text { Forestales y Madera }\end{array}$ & 182 & 528 & $48 \%$ \\
\hline $\begin{array}{c}\text { Manejo Bosque Nativo } \\
\text { Áreas Comerciales }\end{array}$ & 138 & 276 & $28 \%$ \\
\hline Totales & $\mathbf{4 7 2}$ & $\mathbf{1 . 0 0 7}$ & $\mathbf{1 0 0 \%}$ \\
\hline
\end{tabular}

Fuente: Universidad Técnica Federico Santa María [1].

electricidad, ya sea para la industria forestal y/o futuros inversionistas (Tabla 2).

En un estudio reciente, Pontt [1] analiza la factibilidad de desarrollar en el país la capacidad de generar y suministrar energía eléctrica al SIC a partir de las diferentes fuentes de biomasas disponibles. Por ejemplo, desechos provenientes del manejo de plantaciones comerciales (pino, eucalipto, bosque nativo) y residuos generados por la industria forestal y maderera.

En otro estudio, Tokman [2] señala que las energías renovables no convencionales (ERNC) deben jugar un rol cada vez más importante en la diversificación de la matriz energética como estrategia de largo plazo. Ello porque son un recurso autóctono que permite reducir la dependencia externa, disminuir la variabilidad de los costos, y minimizar las emisiones de los gases de efecto invernadero.

El uso de opciones reales para evaluar proyectos de inversión relacionados con energía ha comenzado a desarrollarse en el último tiempo. Rocha, Moreira, Reis y Carvalho [3] aplicaron las opciones reales para evaluar el valor de mercado de las concesiones 
forestales y cuantificaron los beneficios económicos de la ordenación forestal y las políticas regulatorias. Para ello se asume que los precios de la madera siguen dos posibles procesos estocásticos: uno geométrico browniano y otro de reversión a la media.

Siddiqui y Fleten [4] analizan mediante el uso de opciones reales cómo una empresa podría proceder a la comercialización y el despliegue por etapas de distintas tecnologías de energía alternativa. Una nueva tecnología no convencional es una posibilidad, donde se podría llevar a cabo la reducción de costos y medidas de mejora de la producción como un paso intermedio antes de la implementación. Por el contrario, la empresa podría optar por implementar a menor escala la tecnología de energía renovable existente, y utilizar el enfoque de opciones reales para comparar la conveniencia financiera de una u otra decisión.

En este contexto, se requiere evaluar proyectos de generación eléctrica ERNC que demuestren una ganancia equivalente a lo menos a una tasa de libre riesgo.

El presente artículo, a diferencia de otros trabajos, considera la "Metodología de Evaluación Económica de proyectos de inversión a partir de la Teoría de Opciones Reales", pero aplicada a un caso real como es la empresa NSBB, ubicada en San Pedro de la Paz, Concepción, Chile. Esta empresa con capitales noruegos es la principal productora de papel periódico del mundo, con uso intensivo de electricidad y vapor en sus procesos. Por ende, ha sido tomada como una referencia para demostrar cómo aporta este tipo de industria y otras empresas a la matriz energética de las ERNC (energías verdes).

\section{MODELO DE EVALUACIÓN PARA EMPRESA PAPELERA NSBB}

Uno de los mayores problemas que se presenta al realizar el estudio y evaluación económica de proyectos de generación eléctrica es justificar económicamente la inversión de recursos en un proyecto que se enfrenta a condiciones de alta volatilidad e incertidumbre. Esto, debido a que los flujos de caja obtenidos en la práctica probablemente diferirán de los pronosticados por los ejecutivos a cargo de la evaluación. Por lo mismo, al arribar nueva información producto del paso del tiempo, ocurriendo la disipación de la incertidumbre sobre las condiciones de mercado y los montos de los futuros flujos de caja, los ejecutivos tendrán la flexibilidad de alterar la estrategia operativa inicialmente propuesta, para así capitalizar las oportunidades de negocio que se le presenten o para evitar y/o disminuir pérdidas.

Por otra parte, la flexibilidad administrativa de adaptar las acciones futuras de un proyecto, sí puede ser capturada mediante el análisis estratégico, donde la consecución de la ventaja competitiva y el liderazgo de mercado pueden ser medidos en términos de un análisis de la sustentación, flexibilidad y posicionamiento que posee el proyecto, como elementos fundamentales a la hora de evaluar. Sin embargo, su aplicación ha llevado a que condiciones como el juicio e intuición del evaluador cobren importancia, produciendo subjetividad y diferencias al momento de evaluar un mismo proyecto. Este fenómeno ha acelerado la búsqueda y el desarrollo de nuevas técnicas que permitan adecuar la decisión a este tipo de condiciones, surgiendo como una visión alternativa la Teoría de Opciones Reales.

\section{METODOLOGÍA DE OPCIONES REALES PARA UN PROYECTO DE INVERSIÓN}

El artículo [5] plantea que, para incorporar flexibilidad en la evaluación de proyectos de inversión existen hoy dos principales metodologías de evaluación que son el descuento de flujo dinámico y las opciones reales. Al analizar estas metodologías se determinó que los métodos tradicionales no incorporan flexibilidad. El método de descuento de flujo dinámico, por otro lado, sí incorpora la flexibilidad al trabajar con escenarios, el problema aquí es que esos escenarios permanecen estáticos durante el periodo de evaluación, pudiendo llevar a decisiones equivocadas al subvalorar los proyectos de inversión. En el caso del análisis de opciones reales esta metodología sí incorpora la flexibilidad en la evaluación, por lo que sería la más adecuada de utilizar en el caso de tener que trabajar en entornos con alta incertidumbre. En la actualidad se le reconoce a este método su aporte al pensamiento estratégico, ya que cuando se utiliza esta metodología lo primero es determinar las opciones del proyecto para luego valorarlas, por lo que una equivocada determinación de las opciones llevará a una mala valoración. 
En la presente investigación [6] se analiza un enfoque basado en la aplicación de la Teoría de Opciones Reales para realizar la evaluación económica de proyectos de inversión. El mismo, posee el potencial de entregar una aproximación del valor de la flexibilidad que posee un proyecto, permitiendo al evaluador capturar numéricamente el valor de la estrategia. Esto con el fin de presentarla como una herramienta de evaluación alternativa a los métodos tradicionales. La aplicación de esta metodología está basada en el Método Binomial con Transformada Logarítmica.

En relación con el artículo anterior y tesis de estudio del mismo autor, se utilizó la "Metodología de Evaluación Económica de proyectos de inversión a partir de la Teoría de Opciones Reales" [7], la que es presentada en la Figura 1 y considera los siguientes cuatro o seis pasos de aplicación (Figura 1):

Paso 1: Efectuar la evaluación económica del proyecto de inversión mediante la metodología tradicional.

Paso 2: Determinar el VAN del proyecto a partir de los ingresos y costos.

Paso 3: Elaborar diagramas de flujos de caja y política de inversiones.

Paso 4: Generar ideas por medio de la técnica Brainstormingal interior de la empresa para identificar las opciones reales presentes en el proyecto de inversión.

Paso 5: Calcular el valor del proyecto incorporando el valor de las opciones, utilizando el Método Binomial con Transformación Logarítmica.

Paso 6: Analizar resultados obtenidos en los pasos anteriores.

\section{RESULTADOS}

\section{Desarrollo paso 1: evaluación económica} tradicional

La empresa seleccionada en Chile para aplicar la teoría de opciones reales es la empresa NSBB de capitales noruegos. Los principales productos que comercializa son papel periódico, directorio telefónico y otros papeles a base de pulpa mecánica.

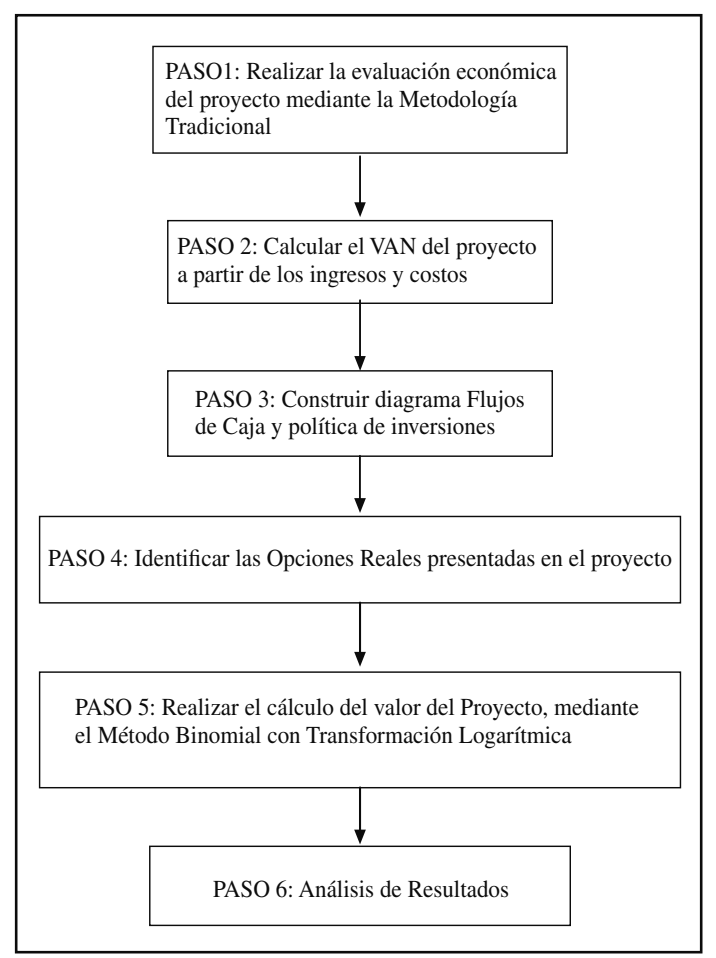

Figura 1. Metodología propuesta.

Fuente: I. Garrido y A. Andalaft [6].

Este tipo de empresa se caracteriza por tener un consumo intensivo de vapor y electricidad en su proceso productivo. Debido a esta situación, se está evaluando la incorporación de una nueva caldera a base de biomasa forestal para la generación de electricidad, con el objeto de aumentar la rentabilidad del negocio y aprovechar las ventajas competitivas al estar relacionada con la industria forestal.

En la Figura 2 se muestra el proceso de la planta de la empresa NSBB, cuyo consumo actual de energía de esta empresa es de $37 \mathrm{MW}$, de los cuales 7 se producen en la planta y los 30 restantes se compran a las compañías generadoras.

En la Figura 3 se observa el proceso considerando la construcción de una nueva caldera de biomasa que permitirá aumentar de 7 a 27 MW la capacidad de producción de energía. Según informó Glenn Rybbert, gerente general de Papeles NSBB, de capitales noruegos, la iniciativa demandará una inversión cercana a los US\$ 60 millones en tecnología y sistemas de manejo para aprovechar al máximo la energía contenida en la biomasa. 


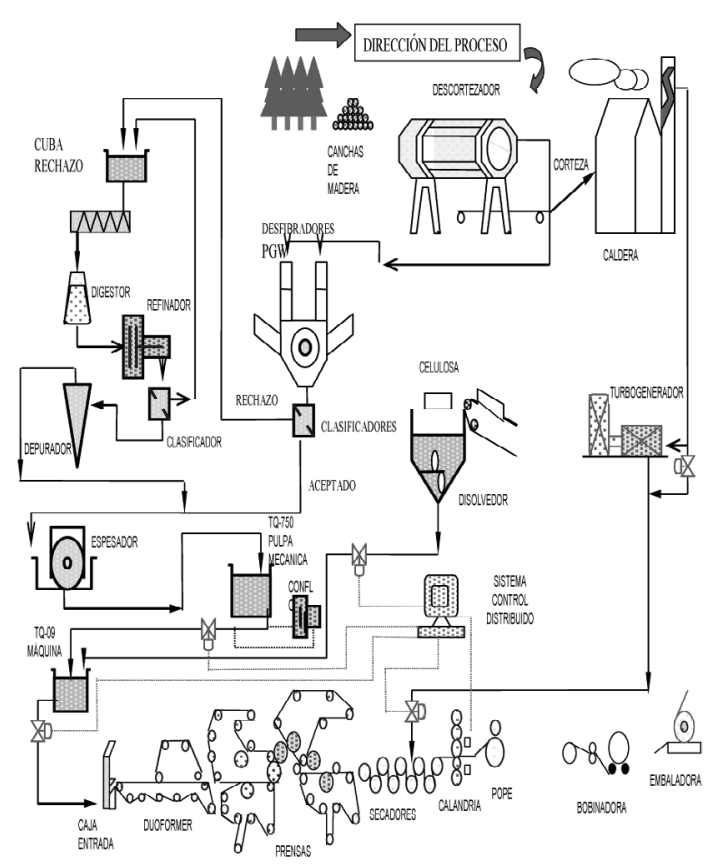

Figura 2. Proceso de planta actual de NSBB. Fuente: Empresa NSBB.

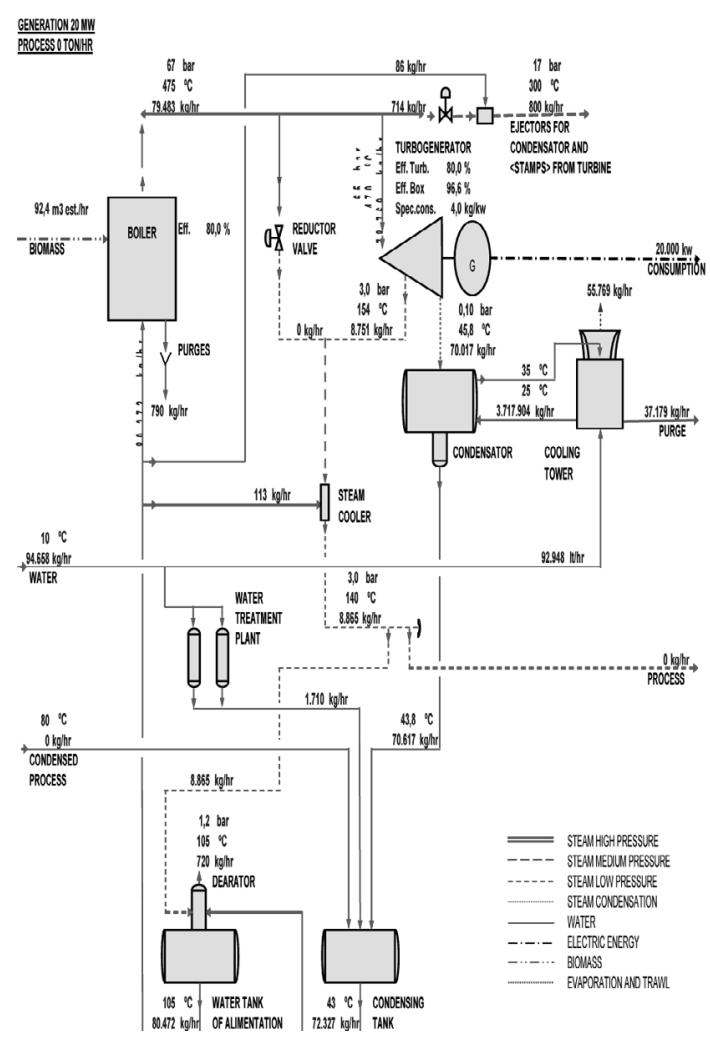

Figura 3. Esquema de planta con caldera nueva con joint venture (para alcanzar los $27 \mathrm{MW}$ ).

Fuente: Empresa NSBB.
Una alternativa de financiamiento que está estudiando la empresa NSBB, para llevar adelante este proyecto es un joint venture (riesgo compartido). Mediante este tipo de cooperación, la empresa NSBB podría obtener parte de su energía eléctrica y vapor para su proceso interno de fabricación de papel. A su vez la empresa socia recibiría biomasa, tratamiento de riles, condensación, así como también podría entregar energía y potencia al Sistema Interconectado Central (SIC $27 \mathrm{MW}$ ), recibiendo a cambio créditos de ERNC (Figura 4). Para ello se requiere que la empresa socia pueda tomar la decisión de invertir en una planta de cogeneración de electricidad con biomasa forestal. Esto solo se haría si se puede conseguir una determinada rentabilidad. Esta planta se ubicaría al interior de los terrenos de la empresa NSBB.

La empresa NSBB no solo está estudiando la posibilidad de un joint venture, sino también se están analizando otras opciones para asegurar la energía eléctrica y vapor que requiere para su proceso de fabricación de papel. Sin embargo, esta información es reservada y estratégica al interior de la empresa, por lo que no se muestran en esta investigación.

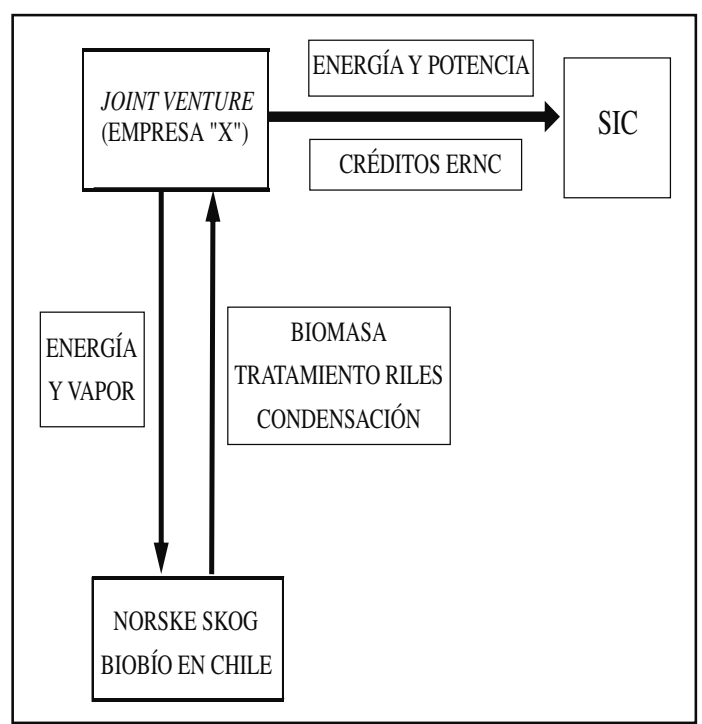

Figura 4. Detalle de intercambios entre empresas. Fuente: Elaboración propia basada en NSBB.

En la Tabla 3 se puede observar una doble sensibilización considerando un alza en el precio de compra de biomasa forestal de $14 \mathrm{US} \$ / \mathrm{m}^{3}$ estéreo y una baja en los ingresos en el primer año de $10 \%$. A continuación en la Tabla 4 se muestran los indicadores tradicionales de la evaluación. 
Tabla 3. Flujo de caja tradicional: joint venture entre NSBB y empresa europea.

\begin{tabular}{|c|c|c|c|}
\hline \multicolumn{4}{|c|}{$\begin{array}{l}\text { PLANTA COGENERACIÓN NUEVA } 20 \text { MW Y ACTUAL } 7 \text { MW (CIFRAS EN } \\
\text { US\$) }\end{array}$} \\
\hline Item / Período (año) & Año 0 & Año 1 & Año 2 al 30 \\
\hline \multicolumn{4}{|l|}{ Prom. Dólar Observado Dic. 2011} \\
\hline \multicolumn{4}{|l|}{ Inversión Inicial: } \\
\hline Estudios & 630.000 & & \\
\hline Costo Equipos & 42.871 .917 & & \\
\hline Costo Montaje & 17.611 .555 & & \\
\hline Capital de Trabajo & 12.222 .694 & & \\
\hline Inversión Total & 73.336.166 & & \\
\hline \multicolumn{4}{|l|}{ Ingresos Operacionales: } \\
\hline \multicolumn{2}{|c|}{ Ingreso por Energía $20 \mathrm{MW}$ a PPA (Contrato Largo Plazo) } & 17.344 .800 & 19.272 .000 \\
\hline $\begin{array}{l}\text { Ingreso por Energía } 7 \text { MW (joint venture } \\
\text { a NSBB) }\end{array}$ & & 3.278 .167 & 3.642 .408 \\
\hline Ingreso por Potencia a Firme (27 MW) & & 1.047 .816 & 1.164 .240 \\
\hline Créditos ERNC & & 2.317 .500 & 2.575 .000 \\
\hline \multicolumn{2}{|c|}{ Ingreso por Vapor (secado papel-joint venture a NSBB) } & 4.355 .100 & 4.839 .000 \\
\hline Ingreso cenizas (venta áridos) & & 2.400 & 24.000 \\
\hline Ingreso Total bruto & & 28.345 .783 & 31.516 .648 \\
\hline \multicolumn{4}{|l|}{ Costos Operacionales: } \\
\hline Pago Peaje & & 180.000 & 180.000 \\
\hline Operaciones & & 720.900 & 810.000 \\
\hline Mantenimiento & & 999.000 & 999.000 \\
\hline Biomasa: $1.400 .000 \mathrm{~m}^{3}$ st/año & & 19.600 .000 & 19.600 .000 \\
\hline \multicolumn{2}{|c|}{ Equipo a cargo de planta (Ingenieros, técnicos y operarios) } & 277.821 & 277.821 \\
\hline Costo Total Operacional & & 21.777.721 & 21.866 .821 \\
\hline Otros Costos: Depreciación & & 2.444 .539 & 2.444 .539 \\
\hline Utilidad Neta Antes de Impuestos & & 4.123 .523 & 7.205 .288 \\
\hline Impuestos (20\%) & & 824.705 & 1.441 .058 \\
\hline Utilidad Neta Después de Impuestos & & 3.298 .819 & 5.764 .230 \\
\hline Depreciación & & 2.444 .539 & 2.444 .539 \\
\hline FLUJO NETO DE CAJA & & $\mathbf{5 . 7 4 3 . 3 5 8}$ & 8.208 .769 \\
\hline
\end{tabular}

Fuente: Elaboración propia basada en NSBB.

Tabla 4. Indicadores tradicionales.

\begin{tabular}{|l|c|}
\hline Indicadores & Resultado \\
\hline VAN (10\%) & 1.805 .917 \\
\hline TIR después de 30 años & $10,3 \%$ \\
\hline Período Recup. Inv. (PRI) & 9 años \\
\hline
\end{tabular}

El periodo normal de recuperación de la inversión corresponde a 7 u 8 años, pero como se aplicó doble sensibilización esta pasó a 9 años. Por otro lado, el precio de compra de la biomasa varía entre 10 y 12 $\mathrm{US} \$ / \mathrm{m}^{3}$ estereo para este tipo de plantas. Sin embargo, se aplicó un precio mayor, debido que en invierno este puede alcanzar los $14 \mathrm{US} \$ / \mathrm{m}^{3}$ estéreo. Este último precio podría bajar dependiendo la gestión de compra (contratos), capacidad de almacenamiento y buen uso de los residuos.
Además, en invierno se requiere mayor biomasa para alcanzar la temperatura deseada en la caldera y producir a igual potencia.

\section{Desarrollo paso 2: calcular nuevo VAN de ingresos y costos}

Tabla 5. Flujo de caja de ingresos y costos.

\begin{tabular}{|c|c|c|c|c|c|}
\hline AÑ̃ & $\begin{array}{l}\text { INGRESOS } \\
\text { US\$ }\end{array}$ & Costos us\$ & \begin{tabular}{|l|} 
FLUJO CAJA \\
ANUAL US\$
\end{tabular} & $\begin{array}{l}\text { FLUJO CAJA } \\
\text { ANUAL } \\
\text { ACUM. US\$ }\end{array}$ & $\begin{array}{c}\text { FLUJO CAJA } \\
\text { ANUAL MM } \\
\text { US\$ }\end{array}$ \\
\hline ANO 0 & 0 & 12.222 .694 & -12.222 .694 & -12.222 .694 & $-12,22$ \\
\hline AÑO 1 & 28.345 .783 & 24.222 .260 & 4.123 .523 & -8.099 .171 & 4,12 \\
\hline AÑO 2 & 516.648 & 24.311 .360 & 7.205 .288 & -893.883 & 7,21 \\
\hline $\mathrm{AN} 03$ & 31.516 .648 & 24.311 .360 & 7.205 .288 & 6.311 .405 & 7,21 \\
\hline AÑ 4 & 31.516 .648 & 24.311 .360 & 7.205 .288 & 13.516 .693 & 7,21 \\
\hline AÑO 5 & 516.648 & 24.311 .360 & 7.205 .288 & 20.721 .981 & 7,21 \\
\hline AÑO 6 & 516.648 & 24.311 .360 & 7.205 .288 & 27.927 .270 & 7,21 \\
\hline AÑO 7 & 31.516 .648 & 24.311 .360 & 7.205 .288 & 35.132 .558 & 7,21 \\
\hline $\begin{array}{l}\text { AÑO } \\
8-29\end{array}$ & 31.516 .648 & 24.311 .360 & 7.205 .288 & 42.337 .846 & 7,21 \\
\hline AÑO 30 & 31.516 .648 & 24.311 .360 & 7.205 .288 & 200.854 .185 & 7,21 \\
\hline TOTAL & 942.328 .575 & 729.251 .696 & 213.076 .879 & & 213,1 \\
\hline \multicolumn{4}{|c|}{ INDICADORES } & \multicolumn{2}{|c|}{ RESULTADO } \\
\hline \multicolumn{4}{|c|}{$\operatorname{VAN}(6 \%$ = tasa libre de riesgo $)$} & \multicolumn{2}{|c|}{84.049 .555} \\
\hline
\end{tabular}

Fuente: Elaboración propia.

En la Tabla 5 se muestran los ingresos y costos del proyecto con un horizonte de evaluación a 30 años, dando como resultado un VAN positivo (tasa libre de riesgo $=6 \%$ ) de US\$ 84.049.555. Este tipo de flujo se diferencia del tradicional al no considerar la inversión como financiamiento propio de la empresa NSBB, sino que la puede conseguir de otras fuentes. Y se aplicó una tasa del $6 \%$ en este flujo para comparar si se obtiene una rentabilidad mayor que invertir en bonos del Tesoro de EE.UU.

\section{Desarrollo paso 3: construir diagramas de flujos de caja}

Flujo de caja tradicional:

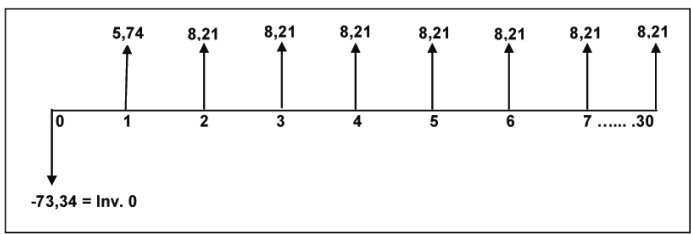

Figura 5. Diagrama de flujo de caja.

Fuente: Elaboración propia.

En este flujo de caja tradicional se puede observar que el resultado del primer año es el más bajo debido 
a los costos de puesta en marcha inicial y producto de que la planta no está produciendo al $100 \%$ de su capacidad de cogeneración de electricidad y vapor. En cambio, a partir del segundo año hacia adelante la empresa NSBB tiene planificado flujos constantes hasta el año 30; con una inversión de MM \$ 73, 34, da como resultado un VAN (10\%) de MM US\$1, 81 (Figura 5).

Otro flujo que se puede analizar es el de ingresos y costos generados por el proyecto, para despejar el efecto de otros egresos, como por ejemplo el impuesto a la renta. Además, en el año cero solo se considera como costo el capital de trabajo para operar en forma normal el primer año. Por tanto, el resultado del VAN con una tasa de descuento de libre riesgo (6\%) es de MM US\$ 84, 05. El VAN mejora debido a que ahora se reducen los costos iniciales al considerar solo el $80 \%$ de generación de electricidad en el primer año por la puesta en marcha (Figura 6).

Flujo de caja de ingresos y costos:

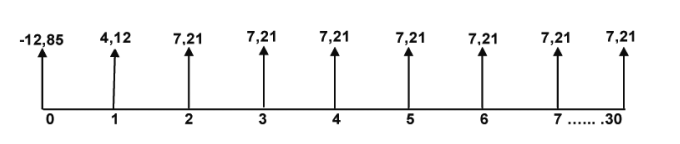

Figura 6. Diagrama de ingresos y costos. Fuente: Elaboración propia.

\section{Desarrollo paso 4: identificar opciones reales en el proyecto de inversión}

Opción 1. Joint venture: En caso de concretarse esta opción real de expansión entre ambas empresas mediante joint venture, se podría conseguir financiamiento para ejecutar el proyecto de la planta de cogeneración de electricidad, con el objeto de obtener un retorno a la inversión por venta de energía y potencia (27 MW), así como también ingresos de créditos ERNC.

Otras opciones: Las otras opciones reales se desconocen por ser información reservada de NSBB, pero se podría plantear como supuesto, en caso de no resultar esta negociación conjunta entre ambas empresas, el rechazo de este proyecto y sustituir la estructura actual de la planta, pero manteniendo la capacidad de generación original de electricidad y vapor (7 MW), siendo necesario suplir los otros dos tercios de electricidad (30 MW) que requiere la planta de fabricación de papel -mediante renovación de contrato con alguna empresa generadora (actual AES Gener).

Por último, se puede comentar que otra opción sería llevar adelante por parte de la empresa NSBB un joint venture con la empresa que actualmente suministra los dos tercios faltantes de electricidad a la planta de fabricación de papel; sin embargo, como supuesto esta empresa no tomaría esta opción, debido a que su estrategia comercial no es una alianza, sino avanzar en el mercado con sus propios recursos y tecnología.

En la Figura 7 se resumen las opciones presentes en el proyecto.

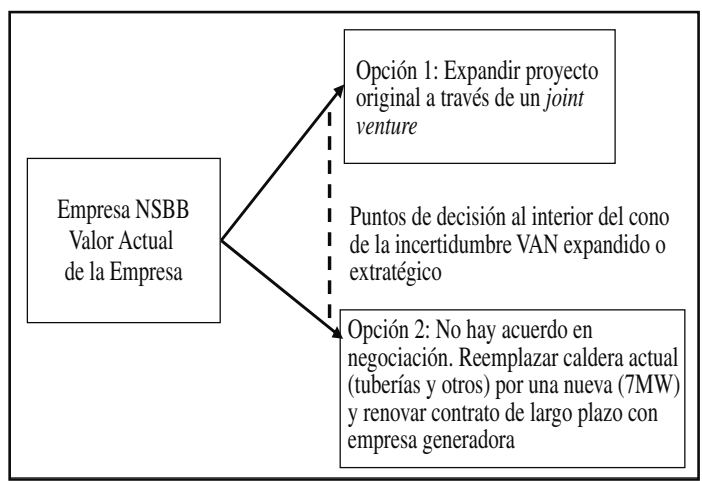

Figura 7. Identificación de opciones reales. Fuente: Elaboración propia.

\section{Desarrollo paso 5: método binomial con transformación logarítmica}

En esta etapa se requiere el ingreso de los parámetros numéricos iniciales para aplicar el modelo $(\mathrm{V}, \mathrm{r}, \sigma$, T, N, I), así como de información relevante sobre las distintas opciones reales a valorar, comenzando con la determinación de los parámetros de entrada.

Tabla 6. Parámetros de entrada.

\begin{tabular}{|l|c|}
\hline \multicolumn{2}{|c|}{ Parámetros de entrada } \\
\hline VAN del Proyecto & 84,05 \\
\hline Tasa libre de riesgo anual & $6 \%$ \\
\hline Varianza de los flujos de caja & 0,32 \\
\hline Horizonte de evaluación (T) & 30 \\
\hline Número de subintervalos & 30 \\
\hline
\end{tabular}

Fuente: Elaboración propia.

La segunda etapa corresponde al proceso de solución binomial con transformada logarítmica, en donde se realiza el cálculo de los parámetros internos del modelo ( $\mathrm{k}, \mu, \mathrm{H}, \mathrm{P})$. 
Tabla 7. Resultados de variables preliminares.

\begin{tabular}{|l|c|}
\hline \multicolumn{2}{|c|}{ Variables preliminares } \\
\hline $\mathrm{K}$ & 0,32 \\
\hline $\mathrm{u}$ & $-0,33$ \\
\hline $\mathrm{H}$ & 0,572 \\
\hline $\mathrm{P}$ & 0,409 \\
\hline $1-\mathrm{p}$ & 0,591 \\
\hline $\mathrm{X}=\mathrm{LN}$ (VAN Proyecto) & 4,431 \\
\hline
\end{tabular}

Fuente: Elaboración propia

$k=\frac{\sigma^{2} T}{N}=\frac{0,32 * 30}{30}=0,32$

$\mu=\left(\frac{r}{\sigma^{2}-\frac{1}{2}}\right)=\left(\frac{0,06}{0,32-0,5}\right)=-0,33$

$H=\sqrt{k+\left(\mu^{*} k\right)^{2}}=\sqrt{0,32+(-0,33 * 0,32)^{2}}=0,572$

$p=\frac{1}{2} *\left(\frac{1+\mu * k}{H}\right)=\frac{1}{2} *\left(\frac{1+(-0,33 * 0,32)}{0,572}\right)=0,409$

$1-p=1-0,409=0,591$

Para efectos del cálculo de los parámetros relevantes se utilizan las siguientes fórmulas, las que son discutidas en el artículo [6] y tesis de investigación [7].

Luego, el programa desarrolla internamente la evolución estocástica del proyecto real en el tiempo. Para esto, realiza en primer lugar la transformación logarítmica del Valor Presente de los Flujos de Caja del Proyecto calculados a partir de los ingresos y costos, generándose la nueva variable $\mathrm{X}$ según $\mathrm{X}=$ $\operatorname{Ln}(\mathrm{V})=\operatorname{Ln}(84,05)=4$, 431. Asimismo, se llevó a cabo la proyección del horizonte de evaluación del proyecto $(\mathrm{T}=30$ años) subdividiendo en $\mathrm{N}=30$ intervalos de largo $\mathrm{k}=0,32$. El programa utilizado es el Excel de Office.

En la tercera etapa se modela la nueva variable $\mathrm{X}$ mediante un proceso estocástico con ascensos y descensos de magnitud $\mathrm{H}=0,572$, realizados al cambiar de estado con probabilidad p y 1-p, respectivamente. Con posterioridad se realiza el proceso iterativo hacia atrás mediante la aplicación del valor presente (operador de descuento - tasa libre de riesgo 6\%), obteniendo el Valor Actual Neto Expandido del proyecto que corresponde a MM US\$ 132, 27 (Figura 8).

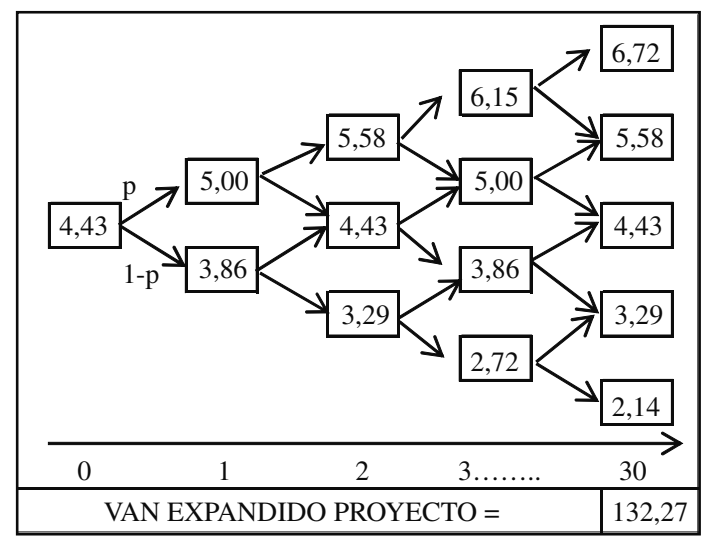

Figura 8. Evolución estocástica del proyecto.

Fuente: Elaboración propia.

\section{Desarrollo paso 6: análisis de resultados}

Tabla 8. Resultados de la opción real de expandir.

\begin{tabular}{|c|c|c|c|c|}
\hline Opción & $\begin{array}{c}\text { VAN } \\
\text { Tradicional } \\
(\mathbf{1 0 \% )}\end{array}$ & $\begin{array}{c}\text { VAN } \\
\text { Expandido } \\
(\mathbf{6 \%})\end{array}$ & $\begin{array}{c}\text { Tipo VAN } \\
\text { Expandido }\end{array}$ & $\begin{array}{c}\text { Valor de } \\
\text { la opción }\end{array}$ \\
\hline \multirow{2}{*}{ Expansión } & 1,81 & 84,05 & Ingresos y costos & 82,24 \\
\cline { 2 - 5 } & 1,81 & 132,27 & $\begin{array}{c}\text { Valor presente } \\
\text { de evolución } \\
\text { estocástica }\end{array}$ & 130,46 \\
\hline
\end{tabular}

Fuente: Elaboración propia.

De acuerdo con la Tabla 6, se puede observar un VAN tradicional de MM\$ 1, 81 (US\$ 1.805.917, equivalente a $\$ 928.241 .444$ ) al considerar una doble sensibilización. Primero, una baja en los ingresos en el primer año en $10 \%$ (puesta en marcha) y, en segundo lugar, un aumento en el precio de compra de la biomasa forestal en $20 \%$.

El aumento en el precio de la biomasa se debe principalmente a la incertidumbre asociado a este proyecto cuando se usa un horizonte de evaluación de 30 años. Además, este recurso es estratégico, no solo para la generación de electricidad y vapor, sino también por el abastecimiento de troncos de madera (pulpa) requerido. En el caso del agua, la empresa utiliza agua de punteras y del río Biobío.

El VAN expandido y que incluye solo los ingresos y costos del proyecto es de MM US\$ 84, 05 (US\$ 84.049.555), equivalente a \$ 43.201.471.163), el que se restó con el VAN tradicional, dando un valor de opción positiva de MM US\$ 82, 24 (US\$ 82.239.555, equivalente a \$ 42.271.131.163). 
Al cálculo del VAN (ingresos y costos) anterior se le aplicó el método binomial con transformación logarítmica por medio del programa computacional Excel, para observar la evolución estocástica de este valor en un árbol binomial a 30 años, según vida útil de proyecto. Posteriormente, estos valores se llevarán a valor presente "hacia atrás" dando un VAN de MM US\$132, 27 (US\$ 132.265.329, equivalente a \$ 67.984.379.009). Este valor se restó con el VAN tradicional resultando un valor de opción positiva de MM US\$ 130, 46(US\$ 130.455.329, equivalente a $\$ 67.054 .039 .009)$.

En consecuencia, se puede derivar del análisis que la opción real de expandir a mediante un joint venture es una buena alternativa para la empresa NSBB en caso de concretarse la negociación con la empresa socia. Sin embargo, también está el supuesto que no resulte el acuerdo, y NSBB tendría que tomar la opción original de cambiar parte de la estructura de la planta de cogeneración de electricidad (tuberías e instalaciones) para mantener la misma capacidad de generación de electricidad (7 MW, equivalente a $1 / 3$ de lo requerido por la fábrica) y vapor para el proceso interno de la fabricación de papel. Complementando esta idea, se requiere negociar el contrato de suministro faltante de electricidad (30 MW) con una empresa de generación.

\section{CONCLUSIONES}

Los métodos tradicionales de evaluación económica de proyectos no son capaces de capturar adecuadamente la flexibilidad intrínseca de los proyectos, mostrando una clara deficiencia a la hora de ser utilizados para realizar la toma de decisiones estratégicas. Por otro lado, las técnicas de valoración de Opciones Reales utilizadas inicialmente en activos financieros han sido aplicadas con singular éxito en la evaluación de proyectos de inversión, presentándose como una alternativa a los métodos de evaluación tradicionales, pues incluyen tanto aspectos estratégicos como de la teoría financiera.

A continuación se presentan los resultados obtenidos en el desarrollo de la aplicación, que para este caso, el valor del proyecto calculado al considerar las Opciones Reales es diametralmente distinto del valor obtenido por el método tradicional.
El VAN tradicional del proyecto con joint venture es positivo por un monto de US\$ 1.805 .917 , a pesar de haber aplicado una doble sensibilización con una baja en los ingresos en el primer año de $10 \%$ por la puesta en marcha en la generación de electricidad, además, considerando la variabilidad del ingreso por venta de electricidad mediante contrato a largo plazo con clientes libres (parámetro de precio US\$ 110 , según comportamiento de mercado y variación del dólar) y un aumento en el costo de la compra de la biomasa forestal de $20 \%$ durante todo el horizonte del proyecto.

Respecto del VAN expandido incluyendo solo ingresos y costos del proyecto, se obtuvo un resultado de US\$ 83.599.910, contra un VAN tradicional de US\$1.805.917. Esta variación ocurre debido al valor de la flexibilidad del proyecto, medida a partir de las Opciones Reales presentes en este, cuyo valor alcanza los US\$ 81.789.910.

De acuerdo con el cálculo del VAN (ingresos y costos) anterior, se le aplicó el método binomial con transformación logarítmica por medio del programa computacional Excel, para observar la evolución estocástica de este valor en un árbol binomial a 30 años, según vida útil de proyecto. Posteriormente, estos valores se llevarán a valor presente "hacia atrás", dando un VAN de MM US\$132, 27 (US\$ 132.265.329, equivalente a \$67.984.379.009). Este valor se restó con el VAN tradicional resultando un valor de opción positiva de MM US\$ 130, 46(US\$ 130.455.329, equivalente a $\$ 67.054 .039 .009)$.

Por tanto, se recomienda la aprobación de este proyecto incorporando la Opción Real de Expandir a través de un joint venture entre la empresa NSBB y futuro inversionista. Esto se ve reflejado en los resultados positivos obtenidos tanto para el VAN expandido del proyecto como para el valor de la opción para el caso analizado. Sin embargo, también está el supuesto que no llegara a buen término este acuerdo conjunto entre ambas empresas, y NSBB tendría que tomar la opción original de cambiar la estructura actual de la planta de cogeneración con biomasa forestal para mantener la misma capacidad de electricidad ( $7 \mathrm{MW}$, equivalente a $1 / 3$ de lo requerido por la fábrica) y vapor para el proceso interno de la fabricación de papel. 


\section{AGRADECIMIENTOS}

El autor agradece a CONICYT por su apoyo financiero mediante la otorgación de la "Beca de Magíster para Funcionarios del Sector Público".

\section{REFERENCIAS}

[1] C. Pontt. "Estudio de Contribución de las ERNC al SIC 2025". Informe Sectorial Final sobre Potencial de la Biomasa para la Generación Eléctrica en Chile al 2025". Investigación realizada por Universidad Técnica Federico Santa María, pp. 1-74. Julio 2008.

[2] M. Tokman. "Nuestros Desafíos Energéticos". Expansiva. Editada por Daniela Crovetto, pp. 1-27. 2010. ISSN 0717-9987. URL: http://www.expansiva.cl/media/en_foco/ documentos/23082010090348.pdf. Fecha de consulta: Julio 2011.

[3] K. Rocha, A.R.B. Moreira, E.J. Reis and L. Carvalho."The market value of forest concessions in the Brazilian Amazon: a
Real Option approach". Forest Policy and Economics. Vol. 8, Issue 2, pp. 149-160. March 2006.

[4] A. Siddiqui and S.E. Fleten. "How to proceed with competing alternative energy technologies: A real options analysis". Energy Economics. Vol. 32, pp. 817-830. 2010.

[5] M. Gallardo y A. Andalaft. "Análisis de la incorporación de flexibilidad en la evaluación de proyectos de inversión utilizando opciones reales y descuento de flujos dinámico". Horizontes empresariales. Vol. $7 \mathrm{~N}^{\circ} 1$, pp. 41-56. Mayo 2008. ISSN: 0717-9901.

[6] I. Garrido y A. Andalaft. "Evaluación económica de proyectos de inversión basada en la teoría de opciones reales". Revista Ingeniería Industrial. Año $2 \mathrm{~N}^{\circ} 1$, pp. 83-89, segundo semestre 2003. ISSN: 0717-9103.

[7] M. Gallardo y A. Andalaft. "Análisis y estudio de metodología de opciones reales para la evaluación comparada de proyectos de inversión". Tesis para optar al grado de Magíster en Ingeniería Industrial. Universidad de Concepción. Concepción, Chile. 2003. 